BULL. AUSTRAL. MATH. SOC.

VOL. 36 (1987) 113-119

\title{
A COMMUTATIVITY CRITERION FOR PRESPECTRAL OPERATORS
}

\author{
WERNER RICKER
}

It is shown that if a bounded linear operator $A$ commutes with a prespectral operator $T$ of class $\Gamma$, then $A$ commutes with the resolution of the identity of class $\Gamma$ for $T$, say $P(\cdot)$, if and only if $A^{*}(\Gamma) \subseteq\left[P^{C}\right]^{*} \Gamma$. Here $A^{*}$ is the dual operator of $A$ and $\left[P^{c}\right]^{*} \Gamma$ is the linear span of the set $\left\{U^{*} \xi ; U \in P(\cdot)^{c}, \xi \in \Gamma\right\}$ where $P(\cdot)^{c}$ denotes the commutant of the range of $P(\cdot)$.

One of the fundamental results in the theory of spectral operators is the commutativity theorem: a bounded operator commutes with a spectral operator if and only if it commutes with $i$ ts resolution of the identity [1; Theorem 6.6]. This commutativity result is known to fail for prespectral operators. Indeed, U. Fixman showed that there exist on $l^{\infty}$ a prespectral operator $T$ with a resolution of the identity $P(\cdot)$ of class $\Gamma=\ell^{1}$ and a bounded operator $A$ which commutes with $T$ but not with every value of $P(\cdot)[1 ; \mathrm{p} .144]$. The crucial point in this example is that $\Gamma$ is not mapped into $\left[P^{C}\right]^{*} \Gamma$ by the dual operator $A^{*}$ of $A$.

Received 4 September 1986. The support of a Queen Elizabeth II Fellowship is gratefully acknowledged.

Copyright Clearance Centre, Inc. Serial-fee code: 0004-9727/87 $\$ A 2.00+0.00$. 
Here $\left[P^{c}\right] * \Gamma$ is the linear span of $\left\{U^{*} \xi ; U \in P(\cdot)^{c}, \xi \in \Gamma\right\}$ and $P(\cdot)^{c}$ denotes the commutant of the range of $P(\cdot)$. The purpose of this note is to establish the fact that if a bounded operator $A$ commutes with a prespectral operator $T$ of class $\Gamma$, then $A$ commutes with the resolution of the identity of class $\Gamma$ for $T$, say $P(\cdot)$, if and only if $A^{*}(\Gamma) \subseteq\left[P^{C}\right] * \Gamma$.

If $X$ is a Banach space, then $L(X)$ denotes the space of all continuous linear operators of $X$ into itself. The identity operator is denoted by $I$. The space of all continuous linear functionals on $X$ is denoted by $X^{*}$. Let $\mathbb{C}$ denote the complex number field and $B$ the o-algebra of Borel subsets of $\mathbb{C}$.

Let $\Gamma$ be a total subspace of $X^{*}$. A set function $P: B \rightarrow L(X)$ is called a spectral measure of class $\Gamma$ if and only if

(i) $\quad P(\mathbb{C})=I$,

(ii) $\quad P(E \cap F)=P(E) P(E)$ for all $E, F \in B$, and

(iii) for all $x \in X$ and all $\xi \in \Gamma$ the $\mathbb{C}$-valued set function $\langle P(\cdot) x, \xi\rangle$ is countably additive on $B$.

It is usually assumed, in addition, that $\sup \{\|P(E)\| ; E \in B\}$ is finite, but this already follows from the requirements (i)-(iii) [2; p.150]. Since $I$ belongs to the range of $P(\cdot)$ it is clear that $\Gamma \subseteq\left[P^{c}\right] * \Gamma \subseteq X^{*}$.

LEMMA 1. Let $P: B \rightarrow L(X)$ be a spectral measure of class $\Gamma$. Then $P(\cdot)$ is also a spectral measure of class $\Lambda=\left[P^{c}\right]^{*} \Gamma$.

Proof. Let $x \in X$ and $\xi \in \Lambda$. Then $\xi=\sum_{r=1}^{n} U_{r}^{*} \xi_{r}$ for some $U_{r} \in P(\cdot)^{c}$ and $\xi_{r} \in \Gamma, r=1, \ldots, n$. It follows that

$$
\langle P(E) x, \xi\rangle=\sum_{r=1}^{n}\left\langle P(E) x, U_{r}^{*} \xi_{r}\right\rangle=\sum_{r=1}^{n}\left\langle U_{r} P(E) x, \xi_{r}\right\rangle=\sum_{r=1}^{n}\left\langle P(E) U_{r} x, \xi_{r}\right\rangle,
$$

for each $E \in B$. Accordingly, $\langle P(\cdot) x, \xi\rangle$ is countably additive.

An operator $T \in L(X)$ is called a prespectral operator of class $\Gamma$ 
if there is a spectral measure $P(\cdot)$ of class $r$, necessarily unique [1; Theorem 5.13], such that $T \in P(\cdot)^{C}$ and the spectrum of the restriction of $T$ to each closed invariant subspace $P(E) X, E \in B$, is contained in the closure of $E$ in $\mathbb{L}$. The measure $P($.$) is called the$ resolution of the identity of class $\Gamma$ for $T$. Spectral operators correspond to the case when $\Gamma=X^{*}[1$; Theorem 6.5]. An example of a prespectral operator (of some class $\Gamma$ ) which is not a spectral operator is given by $T f=g, f \in X=L^{\infty}([0,1])$, where $g(s)=s f(s), s \in[0,1]$, and $\Gamma=L^{1}([0,1])$.

PROPOSITION 1. Let $T \in L(X)$ be a prespectral operation of class $\Gamma$ and $P(\cdot)$ be its resolution of the identity of class $\Gamma$. Then $T$ is also a prespectral operator of class $\Lambda=\left[P^{C}\right]^{*} \Gamma$ with the same $P(\cdot)$ being its resolution of the identity of class $\Lambda$.

Proof. It follows from Lemma 1 that $P(\cdot)$ is a spectral measure of class $\Lambda$ which also satisfies, if considered as being a class $\Lambda$ rather than class $\Gamma$, the properties $T \in P(\cdot)^{C}$ and the spectrum of the restriction of $T$ to each closed invariant subspace $P(E) X, E \in B$ is contained in the closure of $E$. Accordingly, $P($.$) is a resolution of$ the identity of class $\Lambda$ for $T$ and so is the resolution of the identity of class $\Lambda$ for $T$ [1; Theorem 5.13].

If $T \in L(X)$ is a prespectral operator of class $\Gamma$ with resolution of the identity of class $\Gamma$, say $P(\cdot)$, and $A \in L(X)$ commutes with $T$, then it is known that

$$
A\left(\int_{\sigma(T)} f d P\right)=\left(\int_{\sigma(T)} f d P\right) A, \quad f \in C(\sigma(T)),
$$

where $\sigma(T)$ is the spectrum of $T$ [1; Theorem 5.12]. The 'integral' is defined via a process of continuous extension from the $B$-simple functions $[1 ;$ p.120].

The main result can now be published.

THEOREM 1. Let $T \in L(X)$ be a prespectral operator of class $\Gamma$ and $A \in L(X)$ commute with $T$. If $P($.$) is the resolution of the$ 
identity of class $\Gamma$ for $T$, then $A$ commites with each value of $P(\cdot)$ if and only if $A^{*}(\Gamma) \subseteq\left[P^{C}\right]^{*} \Gamma$.

Proof. If $A$ commutes with each value of $P(\cdot)$, then $A \in P(\cdot)^{c}$ and hence, $A *(\Gamma) \subseteq\left[P^{c}\right] * \Gamma$ by definition of $\left[P^{c}\right]^{*} \Gamma$.

Conversely, suppose that $A^{*}(\Gamma) \subseteq\left[P^{c}\right]^{*} \Gamma$. Fix $x \in X$ and $\xi \in \Gamma$. Define $\mathscr{L}$-valued set functions $\mu$ and $\nu$ on $B$ by $\nu(\cdot)=\langle P(\cdot) A x, \xi\rangle$ and $\mu(\cdot)=\langle A P(\cdot) x, \xi\rangle=\left\langle P(\cdot) x, A^{*} \xi\right\rangle$. Then $\nu$ is $\sigma$-additive by definition of $P($.$) being a spectral measure of class \Gamma$ and $\mu$ is o-additive by the hypothesis $A^{*} \xi \in\left[P^{c}\right]^{*} \Gamma$ and Lemma 1 . Since $\mu$ and $\nu$ are regular it follows from (1) that

$$
\int_{\sigma(T)} f d \nu=\int_{\sigma(T)} f d \mu, \quad f \in C(\sigma(T)),
$$

and so the Riesz representation theorem implies that $\nu=\mu$. Since $x \in X$ and $\xi \in \Gamma$ are arbitrary it follows from the totality of $\Gamma$ that $A P(E)=P(E) A$ for each $E \in B$.

COROLLARY 1.1. Let $X$ be a Banach space and $T \in L\left(X^{*}\right)$ be a prespectral operator of class $X$. If $A \in L(X)$ satisfies $A^{*} T=T A^{*}$, then $A^{*}$ conmutes with the resolution of the identity of class $X$ for $T$.

The difficulty with Theorem 1 is that to apply it in practice it is necessary to be able to identify the subspace $\left[P^{c}\right]^{*} \Gamma$ which, in turn, requires a specific knowledge of the resolution of the identity of class $\Gamma$ for $T$, say $P(\cdot)$, and its commutant $P(\cdot)^{c}$. However, it is clear that if $\Gamma$ itself happens to be an invariant subspace of $A^{*}$, then certainly $A$ commutes with $P(\cdot)$. This sufficient condition, although more stringent than the hypothesis $A^{*}(\Gamma) \subseteq\left[P^{c}\right]^{*} \Gamma$ and hence less likely to be satisfied, nevertheless has the advantage that it is easier to verify. Actually, under some reasonable topological assumptions it turns out that the containment $A^{*}(\Gamma) \subseteq \Gamma$ is not too far from the condition $A^{*}(\Gamma) \subseteq\left[P^{c}\right]^{*} \Gamma$. 
PROPOSITION 2. Let $\Gamma$ be a total subspace of $X^{*}$ such that $\Gamma$ is sequentially closed for the weak topology $\sigma(\Gamma, X)$ induced by the dual pairing $\langle\Gamma, X\rangle$. If $P: B \rightarrow L(X)$ is a spectral measure of class $\Gamma$ such that each operator $P(E), E \in B$, is continuous from $(X, \sigma(X, \Gamma))$ into $(X, \sigma(X, \Gamma))$, then $\Gamma$ coincides with the linear span $[U(P)]^{*} \Gamma$, of $\left\{V^{*} \xi ; V \in U(P), \xi \in \Gamma\right\}$ where $U(P)$ denotes the closed algebra generated by $\{P(E) ; E \in B\}$ with respect to the uniform operator topology in $L(X)$.

Remark. An operator $S: X \rightarrow X$ is continuous from $(X, \sigma(X, \Gamma))$ into $(X, \sigma(X, \Gamma))$ if and only if $S^{*}(\Gamma) \subseteq \Gamma$.

Proof. The inclusion $\Gamma \subseteq[U(P)]^{*} \Gamma$ always holds. To show the reverse inclusion it suffices to show, by definition of $[U(P)]^{*} \Gamma$ and the fact that $\Gamma$ is a subspace, that $V^{*} \xi \in \Gamma$ whenever $\xi \in \Gamma$ and $V \in U(P)$. Noting that the range of $P(\cdot)$ is a $\Gamma$ - $\sigma$-complete Boolean algebra in the sense of Definition 2 of [2] it follows from [2; Lemma 2] that if $K$ is the maximal ideal space of $U(P)$, then there exist a spectral measure $Q: B_{K} \rightarrow L(X)$ of class $\Gamma$ and a function $f \in C(K)$ such that $V=\int_{K} f d Q$, where $B_{K}$ is the $\sigma$-algebra of Borel subsets of $K$. In addition, the range of $Q$ coincides with $\{P(E) ; E \in B\}$. Choose a sequence of $B_{K}$-simple functions, say $\left\{f_{n}\right\}$, such that $f_{n} \rightarrow f$ uniformly on $K$. Then $V=\lim \int_{K} f_{n} d Q$, where the limit exists in the uniform operator topology of $L(X) \quad[1 ;$ p.120]. Accordingly (2) $\left\langle x, V^{*} \xi\right\rangle=\langle V x, \xi\rangle=\lim \left\langle\left(\int_{K} f_{n} d Q\right) x, \xi\right\rangle=\lim \left\langle x,\left(\int_{K} f_{n} d Q\right) * \xi\right\rangle$, for each $x \in X$. But, if $g=\sum_{r=1}^{n}{ }^{\alpha}{ }_{r} X_{F(r)}$ is a $B_{K}$-simple function then it follows from the identity $\left(\int_{K} g d Q\right)^{*} \xi=\sum_{r=1}^{n}{ }^{\alpha} Q(F(r))^{*} \xi$, the inclusion $\{Q(F(r))\}_{r=1}^{n} \subseteq\{P(E) ; E \in B\}$ and the assumption that $\Gamma$ is invariant for each operator $P(E) *, E \in B$, that $\left(\int_{K} g d Q\right)^{*} \xi \in \Gamma$. Accordingly, the sequence $\left\{\left(\int_{K} f_{n} d Q\right)^{*} \xi\right\}_{n=1}^{\infty}$ is contalned in $\Gamma$ and, by (2), it converges to $V^{*} \xi$ with respect to the topology $\sigma(\Gamma, X)$. Then 
the $\sigma(\Gamma, X)$-sequential closedness of $\Gamma$ implies that $V^{*} \xi \in \Gamma$.

Remark. It is always the case that $U(P) \subseteq P(\cdot)^{c}$ and hence, under the assumptions of Proposition 2, the subspace $\Gamma$ can be a proper subspace of $\left[P^{c}\right]^{\star} \Gamma$ only if the containment $U(P) \subseteq P(\cdot)^{c}$ is proper. Of course, if it is known for some reason that $U(P)=P(\cdot)^{c}$, then under the assumptions of Proposition 2 it follows that a bounded operator $A$ commuting with a prespectral operator $T$ of class $\Gamma$ (having $P(\cdot)$ as its resolution of the identity of class $\Gamma$ ) commutes with $P($.$) if and$ only if $A^{*}(\Gamma) \subseteq \Gamma$.

Example. Let $X$ be a weakly sequentially complete Banach space and $T \in L(X)$ be a spectral operator with a cyclic vector (that is if $Q: B \rightarrow L(X)$ is the resolution of the identity for $T$, then there exists a vector $x_{0}$ in $X$ such that the linear span of $\left\{Q(E) x_{0} ; E \in B\right\}$ is dense in $X)$. Then $T^{*} \in L\left(X^{*}\right)$ is a prespectral operator of class $X$ with the property that if $A T^{*}=T^{*} A$ for some $A \in L\left(X^{*}\right)$, then $A$ commutes with the resolution of the identity of class $X$ for $T^{*}$ if and only if $A^{*}(X) \subseteq X$. Indeed, with $\Gamma=X$ it follows from [2; Lemma 3] that $P(E)=Q(E)^{*}, E \in B$, is the resolution of the identity of class $\Gamma$ for $T^{*}$. Since $\Gamma$ with the $\sigma\left(\Gamma, X^{*}\right)$ topology is simply $X$ with its weak topology and $P(E)^{*}=Q(E)^{* *}$ has $\Gamma=X \subseteq X^{*}$ as an invariant subspace for each $E \in B$, it suffices to show that $U(P)=P(.)^{C}$ (see Proposition 2 and the Remark following it). But, if $x_{0}^{*} \in X^{*}$ is any Bade functional for $x_{0}$, then $x_{0}^{*}$ is a $\Gamma=X$-cyclic vector for $\left\{P(E)^{*} ; E \in B\right\}$ in the sense of Definition 3 of [2]; see the Remark on page 153 of [2]. Accordingly, the corollary on page 155 of [2] implies that $U(P)=P(\cdot)^{c}$.

For a specific example, let $X=\ell^{1}(I N)$ and $\left\{\lambda_{n}\right\}_{n=1}^{\infty}$ be a bounded sequence in $\mathbb{L}$. Then $X$ is weakly sequentially complete and the operator $T \in L(X)$ defined by $T x=y, x \in X$, where $y_{n}=\lambda_{n} x_{n}, n=1, \ldots$, is a spectral operator with a cyclic vector (for example $x_{0}=\left\{n^{-2}\right\}_{n=1}^{\infty}$ ). 


\section{References}

[1] H.R. Dowson, Spectral theory of Iinear operators, London Math. Soc. Monograph No. 12 (Academic Press, London, 1978).

[2] B. Nagy, On Boolean algebras of projections and prespectral operators, Operator Theory: Advances and Applications Vol. 6 (Birkhauser Verlag, Basel, 1982) 145-162.

Centre for Mathematical Analysis, Australian National University, Canberra,

Australian Capital Territory, 2600 Australia. 